\title{
An Improved Algorithm for Dv-Hop Based on Hops Correction
}

\author{
Yujian Wang ${ }^{1, a}$, Kaiguo Qian ${ }^{2, b}$, Sunyan Hong ${ }^{1, ~ c}$, Chunfen Bu ${ }^{2, d}$ \\ ${ }^{1}$ School of Information and Technology Kunming University, Kunming 650214, China \\ 2 Department of Physics Science and Technology, Kunming University, Kunming 650214, China \\ aemail: 454918730@qq.com, bemail: 769981805@qq.com, cemail: 1390306000@qq.com,
}

demail: 49248139@qq.com

Keywords: Wireless sensor network (WSN); DV - Hop; Hops Correcting; Positioning error

\begin{abstract}
For the disadvantage which is larger positioning error, the RSSI signal strength is used to calculate the hops between the sensors which make it more close to the real network; Meanwhile, the hyperbolic method is adopted to improve the unknown node location which eliminates the cumulative error and improves the positioning accuracy. Simulation experiments show that the positioning error of improved algorithm is better than DV - Hop algorithm that reduced by $15 \%$ or so, and the best case, the positioning error decreases to $15 \%$, meets the demand of sensor network localization accuracy.
\end{abstract}

\section{Introduction}

Wireless sensor networks [1] is a monitoring networks composed by a large number of micro computer equipment which is randomly deployed in task area through wireless self-composition. In most applications, sensor nodes must be sensitive to their own position before particularly elaborating "what a specific event occurs in a location or area" to position and track the external objects [2]. Researchers have put forward some positioning theory and method on the positioning problem of wireless sensor network, which are based on techniques of range-based and range-free [3]. The range-based technique measures the distance or orientation between unknown locations sensors and beacon sensors, and then calculates the position coordinates by trilateration or triangulation. The range-free techniques calculate node position by using the estimated distance or the features of network communication. The positioning performance of range-free algorithm is not as good as that of the range-based algorithm, but the range-free position doesn't require hardware support, and can meet application needs like low cost, low power consumption[4]. Among range-free positioning algorithms, DV-hop [5] algorithm gets widely concern by its simple mechanism that fits the specialty of sensor network. DV-Hop [5-7] algorithm is a classic range-free technique, which calculates average hop distance between beacon nodes, then uses this average hop distance to estimate the distance from unknown nodes the beacon nodes. Next, it completes positioning calculation using least square method or maximum likelihood estimation method .There exist large positioning error and the positioning accuracy cannot meet the positioning performance requirement.

\section{Related Works and DV-Hop}

\section{Related Works}

In DV-hop algorithm, the deployment of physical facilities is unnecessary in distance measurement .Beacon nodes can evaluate the average hop distance of every hop according to the hops of the whole network. The unknown nodes calculates the distance to beacon nodes by evaluating the average hop distance and hop count, and then uses methods of maximum likelihood estimate and least squares to calculate position coordinate of the nodes. Focusing on improving the positioning accuracy of DV-Hop algorithms, researchers have proposed some improved mechanisms. Improved algorithm [8-10] uses methods of evaluating distance weighting correction, 
minimum error positioning and linear programming to reduce positioning error. The method [11] removes the communication link with many errors to improve positioning accuracy. The method [12] corrects the estimated distance, and TLS is used in the positioning to improve positioning accuracy. In evaluating average hop distance among the beacon nodes.

DV - Hop algorithm does not need to deploy a physical device to measure distance, according the beacon nodes to estimate the entire network average hop distance and estimate the distance from nodes to the anchor's distance. Next, it uses the maximum likelihood estimation to locate the node coordinates.

DV - Hop algorithm localization process can be divided into three stages.

(1) Hops between nodes acquisition

Every beacon nodes broadcasts the location information the neighbor node, which includes the serial number of the node ID and location coordinates ( $x, y)$, and the hop parameters initialized value to 1 (hops $=1$ ). Neighbor node receives this data, it will save a copy and broadcasts this data to forward to neighbor nodes. If the receiving node has the data from the same anchor nodes, the smallest hops data is retained, so that, each node containing the data from beacon nodes and the minimum hop count to each beacon.

(2) The average hop distance calculation

Beacon nodes calculate the average network hop distance according to the equation 1 using the distance and hops information between the beacon nodes.

$$
\text { hopzise }_{i}=\frac{\sum_{j=1, j \neq i}^{m} \sqrt{\left(x_{i}-x_{j}\right)^{2}+\left(y_{i}-y_{j}\right)^{2}}}{\sum_{j=1, j \neq i}^{n} h_{i j}}
$$

$h p_{i j}$ is the minimum hops from node $\mathrm{i}$ to node $\mathrm{j}, \mathrm{m}$ is the total number of anchors in the network deployment.

(3) Position to calculate

Beacon node broadcasts the hopzise $e_{i}$ to the unknown node, Through the unknown node store-and-forward way, unknown nodes record the minimum hop count the average distance from to each beacon node, then estimate the distance to beacon node. Least square method is used to calculate its coordinates.

\section{The causes of error analysis}

In the stage of information exchange between nodes, anchor node broadcasts their location information with radius $\mathrm{R}$ distance, then, the hop of the node received the broadcast information becomes 1. As shown in figure 1, within the scope of the anchor node $\mathrm{S}$ communication, node $\mathrm{A}$ and $B$ receive broadcast information from $S$ and count the hops to 1.On the contrary, node $C$ closes the edge of the anchor node $S$, it cannot receive broadcast information from $S$ and hops counting is 2 . In this case, this way of counting error is bigger. Secondly, the unknown node uses the least square method to calculate its own position which introduces the cumulative error. We use the hyperbolic positioning calculation method to eliminate the cumulative error, so as to further reduce the positioning error. 


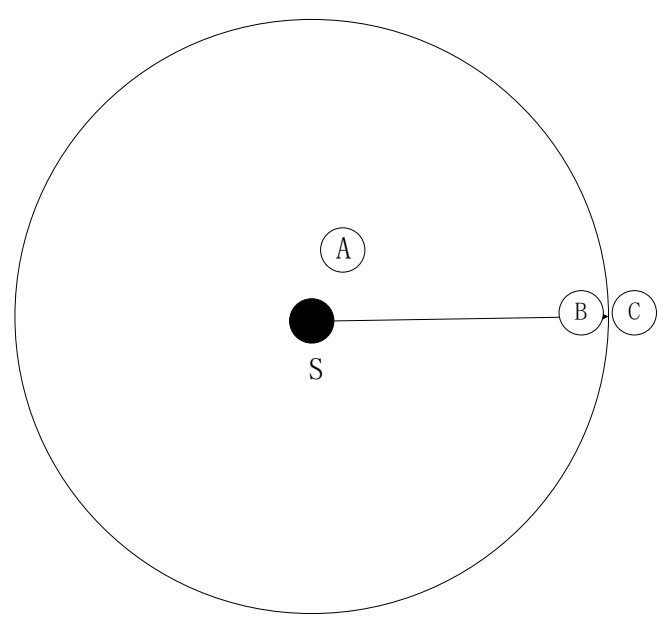

Fig.1. hops error analysis

\section{Localization algorithm based Hop correction}

For the causes of errors for DV - Hop, the node determines the hops according to the RSSI signal intensity values received from the anchor. Anchor calculates average hop distance according to the formula 1 . The unknown node estimates the distance from to the anchor, then using hyperbolic positioning method.

\section{hops correction}

In wireless sensor network node communication uses radio broadcasting, The received signal strength RSSI is function of the transmission power attenuation and transmission distance. Attenuation model [14] is shown in the formula 2.

$$
P L(d)=P L\left(d_{0}\right)-10 n \lg \left(\frac{d}{d_{0}}\right)
$$

$d$ is the distance between the transmitter and receiver, $d_{0}$ is a reference distance, $n$ is the channel attenuation index, $P L(d)$ is signal strength the distance $d$ from transmitter, $P L\left(d_{0}\right)$ is defined according to the hardware specification. Received signal strength indicator.

(RSSI) module is used to analyze the strength of the received signal. D is set to R/2.The corresponding RSSI value is set to signal to determine the threshold $\mathrm{M}$.

When unknown node receives the data packets from the anchor nodes, if the RSSI value is greater than the set signal threshold $\mathrm{M}$, then the node to anchor node hop count hops is 0.5 , if less than the threshold, the hops count to 1.It reduces the hops error for using the RSSI received signal strength decide to hops.

\section{hop distance calculation}

Anchor nodes broadcast their location parameters and hops that is initialized to 0 to neighbor nodes. After receives the packet, neighbor node receiving signal strength analysis module measured RSSI values, and then compare and setting threshold, calculate the hop count information. The node stores a local copy, then broadcasts forwarding, so as to get the whole hops information between nodes. Then anchor nodes calculate the average 1 hop distance, according to the formula 1.

Its calculation of the average jumped from the anchor nodes to flood way to broadcast to the entire network of unknown nodes, using the formula 3 estimate the unknown node to anchor nodes in the distance. Anchor nodes broadcast the average hop distance to the entire unknown nodes by the flood way. The unknown node estimates the distance to anchor nodes using the formula 3.

$$
d_{j i}=\text { hopsize }_{i} \times \text { hops }_{j i}
$$


hops $_{j i}$ is the hops that the unknown node $\mathrm{j}$ to anchor nodes $\mathrm{i}$.

\section{localization calculation}

In positioning of the unknown nodes, it uses hyperbolic positioning method, setting the unknown node $\mathrm{j}$ position $\left(x_{j}, y_{j}\right)$, then choosing the beacon i position $\left(x_{i}, y_{i}\right)$, and the calculation is shown as equation 4.

$$
\left(x_{i}-x_{j}\right)^{2}+\left(y_{i}-y_{j}\right)^{2}=d_{j i}^{2}
$$

If $\mathrm{n}$ beacon nodes participate in positioning, the calculation is shown as equation 5 .

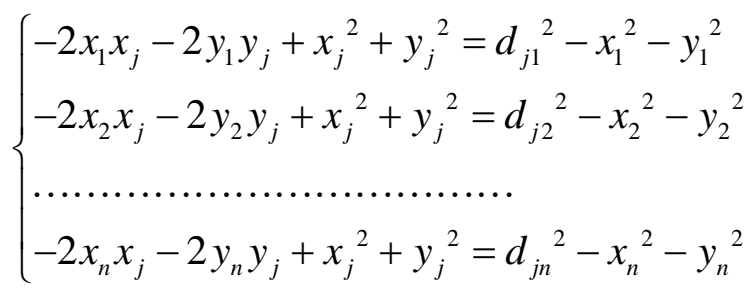

Defined $A_{i}=x_{i}^{2}+y_{i}^{2}, \quad B_{j}=x_{j}^{2}+y_{j}^{2}, Z=\left[x_{j}, y_{j}, B\right]^{T}$

$$
G=\left(\begin{array}{lll}
-2 x_{1} & -2 y_{1} & 1 \\
-2 x_{2} & -2 y_{2} & 1 \\
\vdots & \vdots & \vdots \\
-2 x_{n} & -2 y_{n} & 1
\end{array}\right) \quad H=\left(\begin{array}{l}
d_{j 1}{ }^{2}-A_{1} \\
d_{j 2}{ }^{2}-A_{2} \\
\vdots \\
d_{j n}{ }^{2}-A_{n}
\end{array}\right)
$$

The matrix expression is shown as equation 6 .

$G Z=H$

The calculation is shown as equation 7 .

$Z=\left(G^{T} G\right)^{-1} G H$

Therefore, the coordinate of the unknown node $\mathrm{j}$ is shown as equation 8 .

$\left(x_{j}, y_{j}\right)=(Z(1), Z(2))$

\section{The Experimental Results and Analysis}

In MATLAB R2012a, sensor nodes are randomly distributed in the task area in $1000 \mathrm{~m}^{*} 1000 *$, the topology is shown in figure 2.

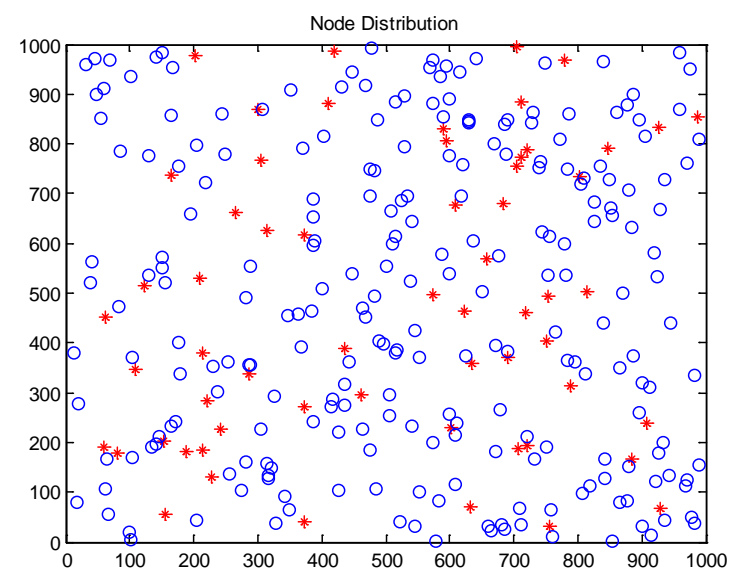

Fig.2. experimental network topology

From the different proportion of anchor and different communication radius, we compare the average relative positioning error for the traditional DV - Hop algorithm, an improved algorithm in 
literature [10] and algorithm in this paper.

The average relative positioning error is defined according to the formula 9.

$E=\frac{\sum_{i} \frac{\sqrt{\left(\hat{x}_{i}-x_{i}\right)^{2}+\left(\hat{y_{i}}-y_{i}\right)}}{r_{-} \text {comm }}}{n}$

In the above equation, $\left(\hat{x_{i}}, \hat{y_{i}}\right)$ is the positioning coordinates of node $\mathrm{i},\left(x_{i}, y_{i}\right)$ is the real coordinate, and $r_{-}$comm is the wireless communication radius of the node.

When setting proportion of the beacon node as $10 \%, 20 \%, 30 \%, 40 \%, 50 \%$ and $60 \%$ and running new algorithm 10 times, the average relative positioning error are shown in Fig.3.

As can be seen from the results, DV - Hop positioning error locates in between $30 \%$ and $34 \%$, the improved algorithm is around $26 \%$, and the improved algorithm in this paper reduces to $15 \%$. Compared with the DV - Hop and the methods of document [10], positioning error in this paper improvement is very obvious, can meet the positioning performance requirements in the sensor network.

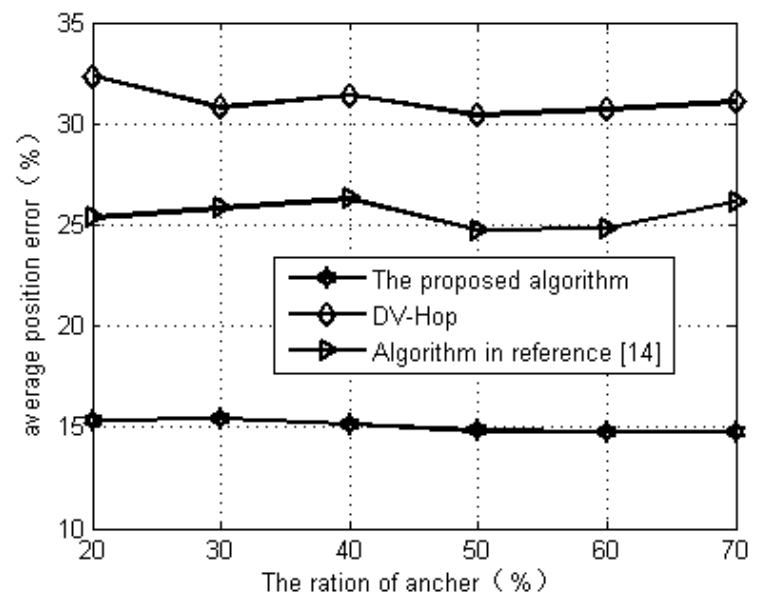

Fig.3. average position error under different anchor node proportion

Fig. 4 is the comparing positioning error results for three algorithms with different communication radius. As can be seen from the results, positioning error in DV - Hop algorithm and this paper algorithm decreases with the increase of communication radius, positioning error in DV - Hop algorithm is reduced from $40 \%$ with radius $150 \mathrm{~m}$ to $30 \%$, literature 10 algorithm maintain

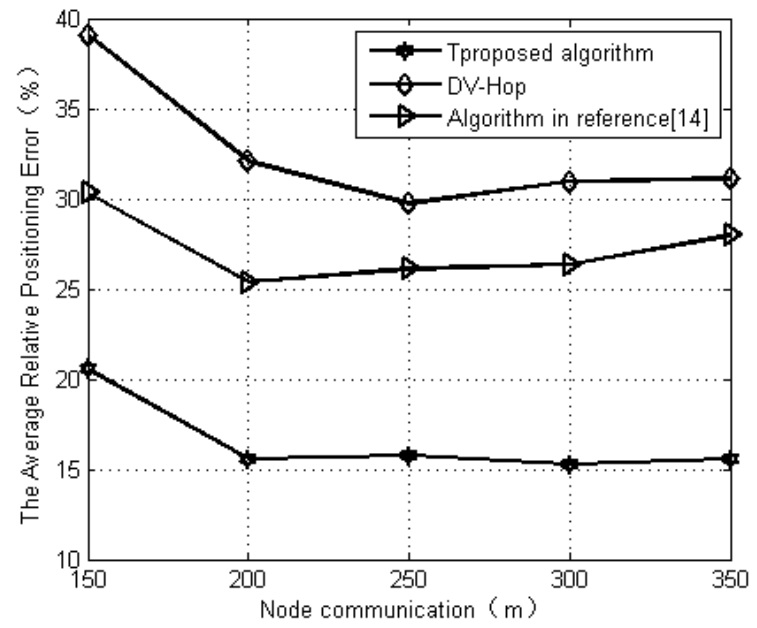

Fig.4. positioning error under different communication radius

$26 \%$.Algorithm in this paper, when communication radius of $150 \mathrm{~m}$, the positioning error is $20 \%$, communication radius is larger than $200 \mathrm{~m}$, the positioning error reduced to $15 \%$ or so, compared 
to the DV - Hop and the algorithm of document [10], positioning error is improved obviously.

Two experiments demonstrate that the algorithm in this paper has smaller positioning error, and has better location performance than DV - Hop algorithm.

\section{Conclusion}

The broadcast radio signal strength RSSI signal is applied to calculate the hops, which make it closer to real the condition, moreover, Hyperbolic locating method is used to eliminate the cumulative error of the least-square method, further reduce the positioning error, improve the positioning accuracy.

\section{Acknowledgement}

In this paper, the research was sponsored by the Kunming University Scientific Research Innovation Team (Project No. 2015CXTD04) and the Open Project of Kunming IOT\&Ubiquitous Engineering Center (Project No. KMIOTKFKT2014003)

\section{References}

[1] Liu Q, Huang X H, and Leng S P. Deployment strategy of wireless sensor networks for Internet of Things[J]. China Communications, 2011, 8(8): 111-120.

[2] WANG F.B,SHI L,REN F.Y. Self-Localization Systems and Algorithms for Wireless Sen-sor Networks. Journal of Software,16(5),pp, 857-867(2005 In chinese)

[3] Asma Mesmoudi , Mohammed Feham, Nabila Labraoui. WIRELESS SENSOR NETWORKS LOCALIZATION ALGORITHMS: A COMPREHENSIVE SURVEY. International Journal of Computer Networks \& Communications (IJCNC) ,2013,5(6):45-64

[4] AN W.X,ZHAO J.M,LI D.A. Research of Localization algorithm for wireless sensor net-works based on Amorphous.Transducer and Microsystem Technologies,32(2),pp,33-35(2013 In chinese)

[5] S. Hou, X. Zhou, and X. Liu, “A Novel DV-Hop Localization Algorithmfor Asymmetry Distributed WSNs,” In 3rd IEEE InternationalConference on Computer Science and Information Technology(ICCSIT), vol. 4, pp. 243-248, 2010.

[6] Y.Y. Li, "Improved DV-Hop Localization Algorithm Based on LocalEstimating and Dynamic Correction in Location for WSNs,"International Journal of Digital Content Technology and itsApplications, vol. 5, issue 8, pp. 196-202, 2011.

[7] Shrawan Kumar, D. K. Lobiyal. Improvement over DV-Hop LocalizationAlgorithm for Wireless Sensor Networks. World Academy of Science,Engineering and Technology,2013

[8] Hou S, Zhou X, andLiu X. A Novel DV-Hop Localization Algorithmfor Asymmetry Dis-tributed WSNs. In 3rd IEEE InternationalConference on Computer Science and Information Technology(ICCSIT), vol. 4, pp. 243-248(2010)

[9] Li Y.Y. Improved DV-Hop Localization Algorithm Based on LocalEstimating and Dynamic Correction in Location for WSNs International Journal of Digital Content Technology and itsApplications, 5(8), pp. 196-202(2011)

[10] Shrawan K, Lobiyal D. K. Improvement over DV-Hop Localization Algorithm for Wireless Sensor Networks. World Academy of Science, Engineering and Technology, 7(4),pp,235-245(2013)

[11] Liu Y, Luo X.Y, Long C.Z. Improved DV-hop Localization Algorithm Based on the Ratio of Distance and Path Length. Journal of Information \& Computational Science, 
9(7),pp1875-1882(2012)

[12] ZHANG J,WU Y.H,SHI F. Localization algorithm based on DV-HOP for w ireless sensor networks. Journal of Computer Applications, 30(2),pp,323-326(2010)

[13] SHI W.R,JIA C.J,LIANG H.H. An Improved DV-Hop Localization A lgorithm for Wire-less Sensor Network. HINESE JOURNALOF SENSORSAND ACTUATORS, 24(1),pp,83-87(2011)

[14] WANG Fu-Bao1, SHI Long, REN Feng-Yuan. Self-Localization Systems and Algorithms for Wireless Sensor Networks. Journal of Software,16(5),pp.857-868,2010 